Philosophie ANTIQUE

\section{Philosophie antique}

Problèmes, Renaissances, Usages

$9 \mid 2009$

Néoplatonisme

\title{
David SEDLEY, Creationism and its Critics in Antiquity
}

Berkeley-Los Angeles-Londres, University of California, 2007 (Sather Classical Lectures 66), xvii + 269 p., ISBN 978-0-520-25364-3.

\section{Gretchen Reydams-Schils}

\section{(2) OpenEdition}

Journals

Édition électronique

URL : https://journals.openedition.org/philosant/2801

DOI : 10.4000/philosant.2801

ISSN : 2648-2789

Éditeur

Éditions Vrin

\section{Édition imprimée}

Date de publication : 13 novembre 2009

Pagination : 211-215

ISBN : 978-2-7574-0124-8

ISSN : 1634-4561

Référence électronique

Gretchen Reydams-Schils, "David sedley, Creationism and its Critics in Antiquity », Philosophie antique [En ligne], 9 | 2009, mis en ligne le 25 juillet 2019, consulté le 03 décembre 2022. URL : http:// journals.openedition.org/philosant/2801; DOI : https://doi.org/10.4000/philosant.2801

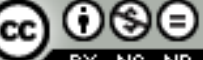

Creative Commons - Attribution - Pas d'Utilisation Commerciale - Pas de Modification 4.0 International - CC BY-NC-ND 4.0

https://creativecommons.org/licenses/by-nc-nd/4.0/ 
Elle prend soin de rappeler la manière dont la pensée de chaque auteur nous est parvenue, l'histoire de sa réception et les principaux débats les concernant. Ses analyses philologiques et stylistiques lui permettent de proposer un grand nombre d'hypothèses nouvelles, comme, par exemple, la détermination de différentes périodes et divers mouvements au sein de ce qui est appelé « le pythagoricisme » (p. 203-206 en particulier). Ce faisant, loin de congédier les témoignages de Platon et d'Aristote, elle définit les positions implicites de ces auteurs par rapport aux pensées archaïques, et montre d'autre part que l'on peut trouver chez Platon des fragments de doctrines de Philolaos.

Ce travail, qui permet de dégager la pensée archaïque dans toute sa complexité et sa diversité, conclut à une certaine unité chez les auteurs archaiques, qui les différencie des auteurs postérieurs. Cette unité tient en particulier à l'indistinction initiale de futures « disciplines », la géométrie, la géographie, l'harmonique, l'astronomie, etc. Le lecteur prend ainsi conscience d'une cohérence et de liens inattendus entre des domaines qui auraient pu paraittre hétérogènes et hermétiques les uns aux autres. Ainsi, le premier chapitre, consacré à Homère, relie l'anatomie à la manière de concevoir les mélodies (p. 45-60). C'est bien la notion d'harmonie qui permet de penser les rapports entre ces deux domaines, parce qu'ils envisagent des parties articulées (mais, chez Homère, il n'y a pas d'unité à proprement parler).

L'indistinction archaïque entre les «disciplines » étaye la définition donnée du schématisme. Les schèmes prennent leur point d'ancrage dans de multiples techniques, en particulier celles de la musique, de la physiologie, de la météorologie, de la cosmologie, de l'anatomie, de la physiologie, de la rhétorique, techniques qui conduisent l'auteur à mener de nombreuses analyses anthropologiques. Plus précisément, les objets techniques (la roue, le navire, l'heptacorde musical) sont, d'Homère à Platon, l'occasion de l'élaboration de notions comme l'infini, le multiple, l'un, et des qualités attachées à l'harmonie. Ces notions restent, d'un bout à l'autre de cette histoire, étroitement liées aux objets techniques. Ainsi, la pensée grecque, qui deviendra à un moment "philosophie » et se constituera en « ontologie », apparaît d'abord être une pensée des techniques.

Séline GÜLGÖNEN

David SEDley, Creationism and its Critics in Antiquity, Berkeley-Los AngelesLondres, University of California Press, 2007 (Sather Classical Lectures, 66), xvii + 269 p. ISBN 978-0-520-25364-3.

Dans cet ouvrage important et original, David Sedley examine les systèmes philosophiques de l'Antiquité qui défendent l'idée d'une cause divine pour l'origine du monde (d'où la notion de creationism) et ceux qui n'admettent pas une intervention de ce genre. Le livre comporte sept chapitres, sur Anaxagore, Empédocle, Socrate, Platon, les atomistes (Démocrite et Épicure), Aristote, et les stoïciens, avec un épilogue sur Galien. Le livre est déjà l'un des plus discutés sur ce sujet, et a ouvert de nouvelles perspectives de recherches.

Dans les deux premiers chapitres, consacrés à Anaxagore et à Empédocle, Sedley s'oppose à la thèse que les présocratiques auraient été des matérialistes, 
sans téléologie développée. D’après Sedley, Anaxagore aurait présenté un système dualiste, avec une intelligence (nous) travaillant sur une matière qui consiste en paires d'opposés sensibles, complètement mélangés (une interprétation que Sedley défend dans un appendice à son chapitre). Le nous crée le monde en séparant par un vortex tout d'abord les éléments, puis, de la terre contenant des semences, toutes les formes de vie. Le nous n'est pas nécessairement incorporel, mais il n'est pas impliqué dans le mélange primordial. L'art auquel on pourrait assimiler le rôle du nous serait celui du fermier. Contrairement à l'interprétation de Platon dans le Phédon (96a-99d), il y aurait une vraie téléologie dans cette vision du monde, ainsi qu'une influence réelle du nous qui expliquerait la nature des choses. Anaxagore ne présenterait pas une perspective religieuse de cette action, mais plutôt une approche scientifique.

Dans le chapitre sur Empédocle, le plus réussi à mon avis, David Sedley tient compte des nouvelles informations contenues dans le papyrus de Strasbourg. Il propose une interprétation selon laquelle, dans le monde tel que nous le vivons actuellement, des phases d'Amour et de Haine alternent (et donc l'univers ne retourne pas au stade d'une sphère parfaite après chaque étape d'influence de l'Amour). Les êtres humains résultent d'une double zoogonie, l'une sous l'influence de l'Amour - mais dans ce cas là il s'agit plutôt d'une race semidivine de démons -, l'autre sous l'influence de la Haine, avec la séparation entre hommes et femmes et la genèse de l'activité sexuelle. Dans les deux phases de l'action de l'Amour, la première, avec la création des organes comme l'œil, fait preuve d'action bienfaisante, téléologique et artistique, tandis que la deuxième procède par une méthode d'expérimentation, avec certains résultats qui ne survivent pas. Cette deuxième étape a des points en commun avec une théorie de l'évolution qui accepte un principe de «convergence », c'est-à-dire la notion que les conditions sont telles que la génération des démons/êtres humains doit se faire. (Dans ce chapitre aussi les points et arguments plus détaillés se trouvent dans des appendices.) Ensemble les deux phases de l'activité de l'Amour présentent une approche combinée de science et de religion.

Dans le troisième chapitre de son ouvrage, Sedley dévalue le rôle de Diogène d'Apollonie dans le développement d'une vision téléologique de l'univers, pour accroitre l'importance de Socrate. Le jeune Socrate qui, dans le Phédon de Platon (96a-99d), se montre insatisfait de la démarcation entre les fonctions de la matière et du nous opérée par Anaxagore, fait place au Socrate qui, d'après Xénophon (Mem. I, 4 et IV, 3) ${ }^{1}$, éprouve le besoin, après l'arrivée des atomistes, de défendre explicitement l'ordre d'un univers créé par un dieu bon. La séparation entre science et théologie aurait stimulé Platon de telle sorte qu'il serait arrivé à une nouvelle vision de la science de la nature dans le Timée, déjà annoncée dans le mythe du Phédon.

1. Passages aussi discutés par A.A. Long, "Socrates in Hellenistic philosophy», Classical Quarterly, 38 (1988), p. 150-171 (absent de la bibliographie). 
En ce qui concerne le Timée, Sedley (en s'appuyant aussi sur le travail récent de Johansen) défend deux thèses contestées ${ }^{2}$. L'une, que le monde tel que Platon le représente dans sa cosmologie n'est pas éternel mais a bien connu un commencement (sur ce point, il est d'accord avec Atticus, Plutarque et d'autres dans la tradition platonicienne, $c f$. p. 107, n. 30). L'autre, que l'univers tel qu'il est constitué par le Démiurge ne montre pas de limitations de ce dernier, c'est-àdire ne contient pas de résidu de forces contraires qui mèneraient au mal. La meilleure argumentation contre un commencement du monde a été développée par M. Baltes ${ }^{3}$, et donc je limite mes remarques ici à la deuxième thèse.

En soutenant que même les manques d'ordre physique et moral font partie du plan du Démiurge et sont donc voulus par lui (p. 119 sq.), Sedley semble sous-estimer les indices dans le Timée de tensions irrésolues dans l'ordre du monde ${ }^{4}$. Il a raison en disant qu'un mot comme ö $\pi \eta \pi \varepsilon \rho$ dans un passage clé (p. 119, n. 57, 56c5 : «dans la mesure où la nécessité le permettait en s'y prêtant volontiers [ scil. à l'intervention du Démiurge] ou en se laissant persuader », trad. Brisson) n'implique pas nécessairement une limitation à l'activité du Démiurge, mais le contexte du passage renforce l'idée d'une limite réelle.

Premièrement, il y a un registre d'expressions pour rendre ces limites $(29 \mathrm{e} 2$, 30a3, 30c7, 32d, 37d2, 38c, 39e, 41c6, 42e2, 48a3, 53b6-7, 65c, $69 \mathrm{~b} 5$ [avec, précisément, ö $\pi \eta], 89 \mathrm{~d}, 72 \mathrm{~d}, 90 \mathrm{c} 2)^{5}$. Comme Sedley lui-même l'admet dans sa conclusion (p. 126 ; cf. aussi p. 228), il doit y avoir une limite ontologique au travail du Démiurge du fait même qu'une copie du monde intelligible telle que notre monde doit toujours être moins parfaite que ce modèle. Mais, précisément, le fait que notre monde ne soit qu'une image présuppose le rôle du réceptacle.

Deuxièmement, il y a le langage de la force et de la violence $(\beta i \alpha: 35 \mathrm{a} 8$, $70 \mathrm{a} 5$; cf. aussi, lié au quatrième point ci-dessous : 43a 7, 64d1, 67a4, 68a1, 81e3, $84 \mathrm{~d} 6,84 \mathrm{e} 6,85 \mathrm{e} 1)$, et pas uniquement la persuasion.

Troisièmement, il y a le fameux dilemme de la construction de la tête qui semble bien indiquer que le pouvoir du Démiurge est restreint $(75 \mathrm{a} 7-76 \mathrm{~d} 3$; cf. aussi un fragment attribué à Chrysippe qui transforme ce passage en évitant un dilemme, SVF II, 1170 = Aulu-Gelle, Nuits Attiques, VII, 1, 7; Sedley nie la différence entre ces deux passages, p. 235).

Quatrièmement, il y a les signes de tensions, de risques de rupture qui se manifestent encore dans le monde tel qu'il a été fabriqué (notamment dans la description de l'entrée d'une âme humaine dans un corps, 42a-d2, 43a-44c4,

2. T.K. Johansen, Plato's Natural Philosophy. A Study of the Timaeus-Critias, Cambridge, Cambridge University Press, 2004.

3. M. Baltes, « Г'́́ Yovev (Platon, Tim. 28B7). Ist die Welt real entstanden oder nicht?», dans K. Algra, P. van der Horst \& D. Runia (éd.), Polybistor: Studies in the History and Historiography of Ancient Philosophy presented to Jaap Mansfeld on bis sixtieth birthday, Leyde, Brill, 1996 (Philosophia antiqua, 72), p. 76-96.

4. Il fait mention, p. 115-116 n. 51 et p. 119 n. 57, de deux des quatre lignes d'argumentation répétées ici, dans G. Reydams-Schils (éd.), Plato's Timaeus as Cultural Icon, Notre Dame, University of Notre Dame Press, 2003, p. 12.

5. Cf. L. Brisson, Platon, Timée/Critias, traduction inédite, introduction et notes, Paris, 1992 (GF, 618), p. 26. 
69c-71d; il y a aussi à expliquer le mélange moins pur qui constitue lâme humaine, 41d6-7, en vue du lien avec des ingrédients «divisibles» $\pi \varepsilon \rho \grave{i} \tau \dot{\alpha}$ $\sigma \omega \dot{\omega} \mu \alpha \alpha, 35 \mathrm{a})$.

Ce problème ne peut être résolu sans une réponse à la question de savoir comment Platon conçoit le rôle du réceptacle, auquel Sedley n'accorde que quelques lignes (p. 116-117). Il est bien connu que Platon semble avoir laissé une ambiguïté profonde dans son exposé, entre, d'une part, un réceptacle neutre, et donc ouvert à toutes les actions ordonnatrices que peut exercer un Démiurge, et, d'autre part, ce même réceptacle conçu comme désordre, avec des traces des éléments en mouvement même «avant» l'intervention du Démiurge. Cela explique que la tradition platonicienne ait eu de grandes difficultés à éviter une lecture dualiste du Timée de Platon ( $c f$. aussi Sénèque Ep. 58, 27-28). Le moins qu'on puisse dire, c'est que la question n'était pas complètement résolue dans le Timée lui-même, et que, d'un point de vue herméneutique, le texte est ouvert à des interprétations divergentes.

Une des conséquences de l'interprétation du Timée par Sedley, c'est qu'il minimise l'écart non seulement entre Socrate et les stoïciens (une thèse généralement acceptée), mais aussi entre ces derniers et Platon (aussi bien que l'Ancienne Académie ${ }^{6}$ ). Mais si l'on suit cette piste, il devient quasi impossible d'articuler la différence cruciale entre une position platonicienne qui considère ce

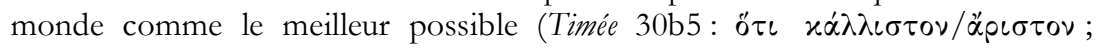
53b6-7), et la fameuse thèse stoïcienne, souvent contestée dans l'Antiquité, qui prétend que le monde est parfait, tout simplement ( $c f$. p. 210 ; 234-238). Cette thèse repose, entre autres, sur le fait que les stoïciens ont tranché l'ambiguité du réceptacle dans le Timée de Platon en faveur d'une matière complètement neutre et donc complètement ouverte à l'activité du principe divin (contra Sedley, qui groupe les deux perspectives ensemble, p. 210). En somme, pour Platon, dans le Timée, le réceptacle est un principe d'imperfection (dont on peut alors minimiser l'effet négatif dans le sens de l'interprétation de Sedley, ou non), ce qui n'est pas du tout le cas pour les stö̈ciens.

Dans la tradition atomiste, telle qu'elle est représentée par Démocrite, Épicure et Lucrèce, nous trouvons le cas le plus clair dans l'Antiquité d'un matérialisme. Cette pensée développe tout un arsenal d'arguments contre une intervention divine dans l'univers (dont Cicéron dans son De Natura Deorum témoigne aussi), et elle arrive à une notion sophistiquée de l'infini et du principe d'isonomia (parité de distribution) pour expliquer le monde comme résultat accidentel de combinaisons d'atomes dans un vide. Mais alors, comment rendre compte des structures régulières dans la nature telle que nous la connaissons, comme, par exemple, le fait qu'un cochon ne peut naître d'un bananier ( $c f$. aussi le chapitre suivant sur Aristote, p. 192)?

6. D.N. Sedley, «The origins of Stoic God», dans D. Frede \& A. Laks (éd.), Traditions of Theology. Studies in Hellenistic Theology, its Background and Aftermath, Leyde, 2002 (Philosophia antiqua, 89), p. 41-83 ; J. Dillon, The Heirs of Plato: A Study of the Old Academy (347-274 BC), Oxford, 2003. 
Dans son traitement d'Aristote (qui se concentre sur le deuxième livre de la Physique), Sedley, comme Johansen avant lui, met l'accent sur les points communs entre celui-ci et Platon. Les innovations les plus importantes introduites par Aristote sont de n'accorder à son dieu suprême aucune responsabilité pour une intervention directe dans le monde considéré comme éternel, et d'attribuer à toute la nature (non seulement aux organismes individuels, mais aussi à l'univers entier) une téléologie radicale. La critique qu'Aristote adresse aussi aux présocratiques s'applique en réalité surtout aux atomistes. Il rejette premièrement leur notion de nécessité matérielle en l'intégrant dans une nécessité conditionnelle. Cependant la nécessité matérielle n’est pas éliminée, et peut donc se manifester et agir contre l'ordre imposé par la forme. (Ici Sedley postule une différence entre Aristote et Platon, tandis qu'on pourrait interpréter cette position comme un autre point de convergence.) Deuxièmement Aristote attaque la thèse que le monde et la nature proviendraient du hasard.

Puisque Sedley attribue déjà tant de thèses de la cosmologie et de la physique stoïcienne au Socrate tel que Xénophon le présente et au Platon du Timée, il se concentre dans son chapitre dédié aux stoïciens sur les fameux syllogismes de Zénon, tels qu'ils sont présentés par Sextus Empiricus dans un passage spécifique $(M .9,88-110)$ qui, en effet, établit un lien entre ces arguments, Xénophon (Mem. I, 4), et le Timée. Il termine son étude par quelques remarques sur Galien (spécifiquement De Usu Partium), qui pousse l'analyse téléologique encore plus loin que Platon et Aristote, rejette la notion judéo-chrétienne selon laquelle un fiat de Dieu serait suffisant comme explication de la nature des choses, et finalement se range du côté du Socrate de Xénophon en rejetant toute spéculation purement théorique dans la science (De Placitis Hippocratis et Platonis IX, 7, 9-16).

Le grand mérite de cette étude, c'est d'avoir démontré la continuité du débat dans la philosophie ancienne sur les origines du monde et sa structure causale. C'est le telos que l'auteur lui-même s'était proposé, et vu sous cet angle, l'ouvrage est un succès éclatant, appelé à constituer l'ouvrage de référence sur la question.

Gretchen REYDAMS-SCHILS

Robert ZABOROWSKI, Sur le sentiment chez les Présocratiques: Contribution psychologique à la philosophie des sentiments, Varsovie, Stakroos, 2008, 256 p.

L'intérêt suscité par le concept d'émotion chez les Grecs a ouvert une voie d'interprétation nouvelle en histoire de la psychologie. D. Konstan a montré en particulier, dans The Emotions of the Ancient Greeks (Toronto, 2006), qu'en accordant à certaines émotions une valeur cognitive, on relativise les lectures qui privilégient une dichotomie entre l'exercice de la pensée rationnelle et la dimension affective, non rationnelle, de la vie psychique. Il reste cependant à montrer comment notre notion moderne d'émotion ou de sentiment parvient à rendre compte de la manière dont les Grecs décrivaient certaines affections. Il faut donc retracer l'histoire de la notion de «sentiment», d'Homère à ses premiers "théoriciens », à savoir Platon et Aristote, dont la typologie des émotions dans la Rhétorique constitue souvent un point de départ interprétatif, et non un point d'arrivée. R. Zaborowski, qui s'était intéressé à certaines affections particulières 